\title{
Time Analysis for Wireless Wake-Up Radio Transceivers
}

\author{
Amir Bannoura, Omar Gorgis, \\ Leonhard Reindl and Christian Schindelhauer ${ }^{*}$
}

Department of Microsystems Engineering, Computer Networks and Telematics*

University of Freiburg

Georges-Köhler-Allee 106

79110, Freiburg, Germany

Email: amir.bannoura@imtek.de

Keywords: wake-up radio transceiver, wireless sensors, time analysis

\begin{abstract}
Energy consumption is the main concern in wireless sensor networks (WSNs). A node's life is prolonged by reducing idle listening and packet overhearing. The energy consumption poses a problem to the life span of a wireless node. A duty cycle approach reduces energy consumption of a node but increases latency of data delivery. The delay the node needs until it switch from receiving mode into transmitting mode can be solved through the use of wake-up receiver, where the node reacts only when it's woken up. This process eliminates the time spent waiting the transmission slot assigned through the scheduling procedure. In this paper, we present the wakeup radio transceiver and analyze the time demand of the wake-up system.
\end{abstract}

\section{Introduction}

The performance of WSNs depends on the connectivity of the individual sensors that are part of the network. Currently, sensor nodes are operated using limited resources. After certain time, a nodes' energy is consumed due to its usage and the most consuming energy source is the radio. A duty cycle approach is used to reduce the energy consumption. This approach reduces the time the node spends on listening to the medium through turning off and on the radio transceiver. Nevertheless, L. Gu et. al. shows that $21 \%$ of the energy is used in really tracking targets, and $7 \%$ is used in sleep mode. However, $72 \%$ of energy is still wasted in a waiting status because the node periodically wakes up to listen to potential wake-up signals, or continuously operates in a low-power stand-by listening mode. [1]. Beside, the node that uses duty cycle approach must wait its slot time when transmitting a packet. The waiting time to process and transmit a packet to another node is an energy waste for that node. According to this, the waiting time and processing must be reduced to a minimum. Due to its efficiency and low energy consumption, the concept of wake-up radio system (WuR) has emerged in which nodes only communicate when it is required. The node is not required to stay tuned and listening to the medium. WuR is considered a benefit in WSNs that prolong the life of the network which provides a continuous functionality through its connectivity. On the other hand, WuR is associated with several 
challenges [4]. The limitation of the wake-up distance provides the need for multihop wake-up to reach to a desired destination. Increasing the number of wake-up hops, increase the end-to-end delay. Our interest is focused on the study of the time required that the transmitter needs to perform the wake-up procedure. The rest of this paper is organized as follows: Section 2 introduces the wake-up radio system; Section 3 presents the features that have a direct impact on wake-up time; Section 4 explains the experiment and the results that we obtained; a conclusion remarks is given in section 5 .

\section{Wake-Up Radio System}

Gamm et. al. [2] developed a (WuR) that combines a microcontroller and Austria Microsystems chip AS3932 [3]. The AS3932 operates as a trigger to activate the microcontroller and drive it from its sleep state where only $2.8 \mu \mathrm{A}$ is required in order to interact with the surrounding environment. The WuR consists of two parts as it appears in figure 1. The upper part is used for demodulating the wake-up signal and the lower part is used for data communication. The two parts are connected to an antenna switch (ADG918) from Analog Devices. The antenna switch is controlled by the microcontroller that facilitates the communication according to the intended use of the system. When the microcontroller is in an active state, the antenna switch is connected to the CC430 [5]. Otherwise, the antenna switch is connected to the demodulation path, when the microcontroller is in a sleep state.

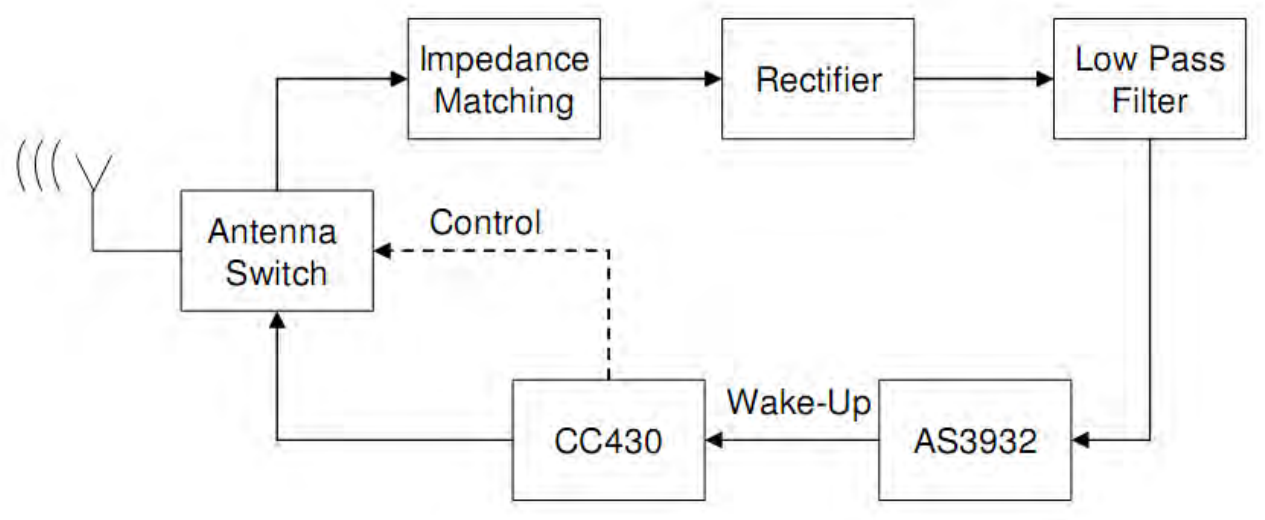

Figure 1: A node schematic

The CC430 has an integrated main transceiver that operates using a frequency of $868 \mathrm{MHz}$. However, the AS3932 uses an Amplitude Shift Keying (ASK) modulation scheme and it is only able to detect a modulated signal with $125 \mathrm{kHz}$. In order to trigger an interrupt to wake-up the microcontroller at the receiver side, a wake-up transmitter (WuTx) sends the $868 \mathrm{MHz}$ carrier frequency by turning it on and off to achieve a square signal that represents a $125 \mathrm{kHz}(\mathrm{On} / \mathrm{Off}$ keying). The AS3932 contains a correlator that is responsible for detecting a bit pattern which indicates the address of the wake-up receiver (WuRx). When the WuRx address matches the received pattern, the AS3932 sends an interrupt to wake-up the microcontroller. In turn, the microcontroller flips the antenna switch and connects it to the microcontroller to make it possible for data exchange. 


\section{WuR Features}

\subsection{Time Demand}

WuR gives an opportunity to reduce energy by being in a sleep state when no communication is required from the node. In order to wake-up a node from a sleep state to active state, a wake-up signal with a specific address needs to be sent. The time the WuTx requires to wake-up WuRx is a crucial for sensors interaction. The WuR replaces conventional schemes like duty cycle in sense that when the node wakes-up, it has the ability to transmit information directly without waiting any certain time assuming the medium is clear. Nevertheless, the procedure to wake-up a node through processing the wake-up signal and transmitting it to a receiver will take a considerable amount of time. Some applications such as monitoring critical infrastructure might need a fast response time. In this case, wake-up time is an important factor that must be reduced for such applications.

\subsection{Wake-Up Distance}

The distance is another major feature of WuR. There is a difference between the distance ranges of wakeup and data exchange communication. When a node is resided at a distance outside the wake-up coverage area, there will be a need for multihop wake-up to wake the intended node. This increases the number of hops for the wake-up. One solution is to extend the wake-up range which eliminates the need for intermediate wake-up nodes. However, the transmission power which is used to transmit the wake-up signal and the design of the antenna are important factors for extending the wake-up distance.

\section{Experimental Results}

We performed an experiment to estimate the time required for wake-up radio at transmitter side to transmit a wake-up signal. We connected the WuTx to a Tektronix (MSO 4104) oscilloscope on two channels; the first channel attached to SMA connector that is connected to the antenna to calculate the time needed for the wake-up signal to be transmitted. The second channel is connected to an output pin. A trigger is set at specific points to generate an impulse signals as in figure 2 . The impulses are used to estimate the processing and preparation time before and after a wake-up signal transmitted to a WuRx.

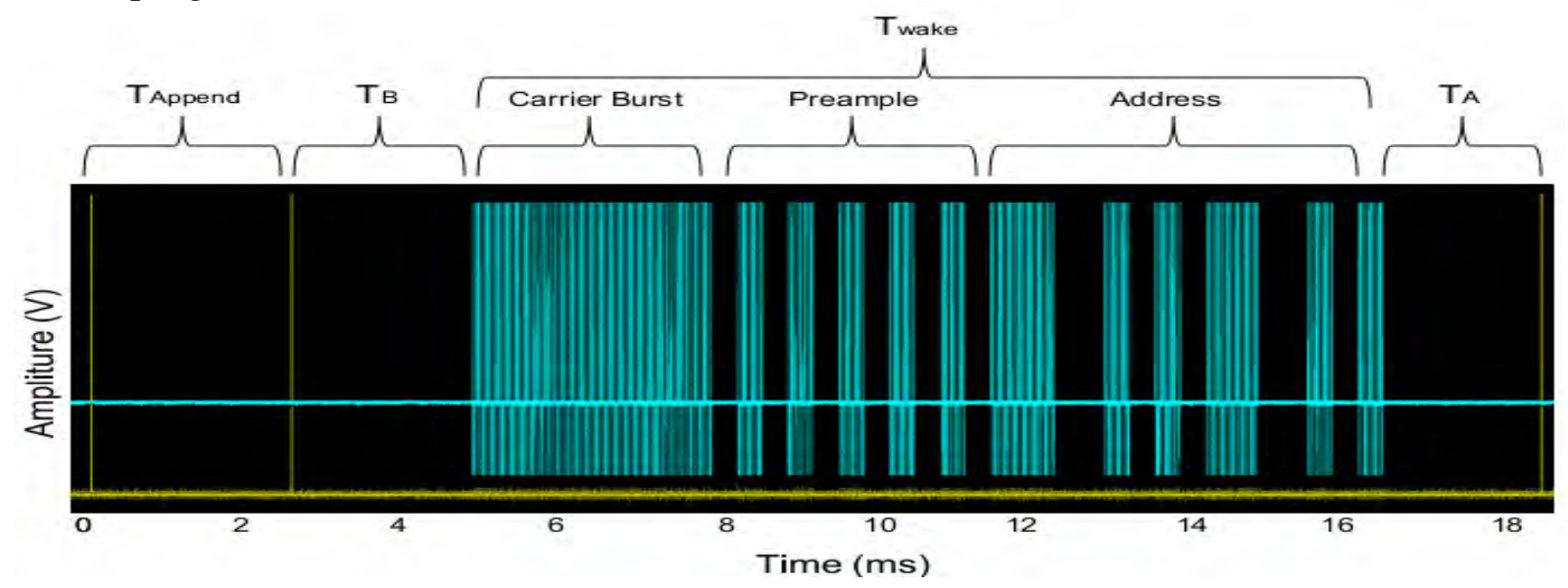

Figure 2: Wake-Up Signal and Triggered Impulses 
The benefit of these triggers is to calculate the required time for the WuTx to switch to receive mode to receive an Ack packet. Otherwise, if the WuRx sends an Ack indicating his readiness to receive data while the WuTx didn't finish processing from the transmission state, the Ack will be missed and the WuTx will think that the WuRx is dead. Figure 2 displays the result that we obtained from processing and transmitting a wake-up signal, where Twake is the time required to transmit the wake-up signal. Тв is the time required for processing and initializing the registers before transmitting the wake-up signal, $\mathrm{TA}_{\mathrm{A}}$ is the time required to adjust the microcontroller registrs after transmitting the wake-up signal and TAppend is the time required to append the address into the wake-up signal before transmitting it. The wake-up signal consists of carrier burst and preamble which are fixed so they are constructed outside the transmission state. On the other hand, the wake-up address is changed dynamically according to the node that we need to wake. The total time required to perform the wake-up process at the WuTx side equals $19.3 \mathrm{~ms}$. Table 1 displays the result of the wake-up time segments.

\begin{tabular}{||c||c|}
\hline \hline Symbol & Time $(\mathrm{ms})$ \\
\hline \hline $\mathrm{T}_{\text {Append }}$ & 2.65 \\
\hline \hline $\mathrm{T}_{\mathrm{B}}$ & 2.36 \\
\hline \hline $\mathrm{T}_{\text {wake }}$ & 12.2 \\
\hline \hline $\mathrm{T}_{\mathrm{A}}$ & 2.09 \\
\hline \hline$\sum$ & 19.3 \\
\hline \hline
\end{tabular}

\section{Conclusion}

Table 1: Wake-Up Time Segments

In this paper, we presented an overall description of the wake-up radio system and explained some of the features that it depends on such as time transmission of a wake-up signal and distance range. We analyzed the required time the wake-up transmitter needs to process and transmit a wake-up signal. We observed that $63.2 \%$ of the time is spent on transmitting the wakeup signal, $13.7 \%$ is spent on preparing which node to wake-up and only $23.1 \%$ is spent on initializing the registers.

\section{References}

1. L. Gu and J.A.Stankovic. Radio-Triggered Wake-Up for Wireless Sensor Networks. RealTime Systems, Springer, Vol. 29, No. 2-3, ISSN: 0922-6443, Mar. 2005, pp 157 - 182.

2. G. Gamm, M. Sippel, M. Kostic, and L. Reindl, "Low power wake-up receiver for wireless sensor nodes," in Proc. IEEE International Conference on Intelligent Sensors, Sensor Networks and Information Processing (ISSNIP'IO), Brisbane, Australia, Dec. 2010.

3. AS3932 3D Low Frequency Wakeup Receiver [online]. Available: http://www.ams.com/eng/Products/RF-Products/Low-Frequency/AS3932

4. Wake-up receivers for wireless sensor networks: benefits and challenges, Ilker Demirkol, Cem Ersoy and Ertan Onur, IEEE Wireless Communications, vol. 16, no. 4, pp. 88-96, Aug. 2009.

5. CC430F5137 16-Bit Ultra-Low-Power MCU [online]. Available: http://www.ti.com/product/cc430f5137 